UDC 517.588, 511.4

V. A. Gorelov

\title{
ON CONTIGUITY RELATIONS FOR GENERALIZED HYPERGEOMETRIC FUNCTIONS
}

\begin{abstract}
We derive formulas that generalize contiguity relations of Gauss hypergeometric functions to the case of hypergeometric functions satisfying differential equations of arbitrary order and also of solution matrices of their corresponding homogeneous differential equations.
\end{abstract}

Key words: generalized hypergeometric functions, contiguous functions, Siegel's method

2010 Mathematical Subject Classification: 33C20, 11J91

1. Introduction. Let $\mathbb{A}$ be the set of all algebraic numbers, $\mathbb{Z}^{+}=\mathbb{N} \cup\{0\}, \quad \mathbb{Z}^{-}=\mathbb{Z} \backslash \mathbb{N}, \quad \delta_{i}^{j}$ be the Kronecker delta, $\mathbb{C}(z)$ be the set of all rational functions with coefficients from $\mathbb{C}, \mathbb{C}\left[z^{ \pm 1}\right]$ be the ring $\mathbb{C}\left[z, z^{-1}\right], \quad M(q, K)$ be the set of all $q \times q$ matrices with elements from a ring $K$ and $G L(q, K)$ be the general linear group in $M(q, K)$.

The functions

$$
{ }_{l} \varphi_{q}(z)={ }_{l} \varphi_{q}(\vec{\nu} ; \vec{\lambda} ; z)={ }_{l+1} F_{q}\left(\begin{array}{c}
1, \nu_{1}, \ldots, \nu_{l} \\
\lambda_{1}, \ldots, \lambda_{q}
\end{array} \mid z\right)=\sum_{n=0}^{\infty} \frac{\left(\nu_{1}\right)_{n} \ldots\left(\nu_{l}\right)_{n}}{\left(\lambda_{1}\right)_{n} \ldots\left(\lambda_{q}\right)_{n}} z^{n}
$$

where $0 \leqslant l \leqslant q,(\nu)_{0}=1,(\nu)_{n}=\nu(\nu+1) \ldots(\nu+n-1), \quad \vec{\lambda} \in\left(\mathbb{C} \backslash \mathbb{Z}^{-}\right)^{q}$, $\vec{\nu}=\left(\nu_{1}, \ldots, \nu_{l}\right) \in \mathbb{C}^{l}$ are called generalized hypergeometric functions (see $[1,2,6,8,9])$.

The function $\varphi_{q}(\vec{\nu} ; \vec{\lambda} ; z)$ satisfies the (generalized) hypergeometric differential equation

$$
L(\vec{\nu} ; \vec{\lambda} ; z) y=\left(\lambda_{1}-1\right) \ldots\left(\lambda_{q}-1\right)
$$

(see [8], Chap. 5, §1, formula (5)), where

$$
L(\vec{\nu} ; \vec{\lambda} ; z) \equiv \prod_{j=1}^{q}\left(\delta+\lambda_{j}-1\right)-z \prod_{k=1}^{l}\left(\delta+\nu_{k}\right), \quad \delta=z \frac{d}{d z} .
$$

(c) Petrozavodsk State University, 2018 
The properties of the functions

$$
{ }_{l} F_{q-1}(\vec{\nu} ; \vec{\lambda} ; z)={ }_{l} F_{q-1}\left(\begin{array}{c}
\nu_{1}, \ldots, \nu_{l} \\
\lambda_{2}, \ldots, \lambda_{q}
\end{array} \mid z\right)=\sum_{n=0}^{\infty} \frac{\left(\nu_{1}\right)_{n} \ldots\left(\nu_{l}\right)_{n}}{n !\left(\lambda_{2}\right)_{n} \ldots\left(\lambda_{q}\right)_{n}} z^{n}
$$

satisfying homogeneous linear differential equations were studied most minutely. We assume that the vector $\vec{\lambda}$ related to the function ${ }_{l} F_{q-1}(\vec{\nu} ; \vec{\lambda} ; z)$ contains a component equal to 1 , and this component is automatically moved to the first position.

A lot of functions used in mathematics are hypergeometric $\left(e^{z}\right.$, Gauss hypergeometric functions, Kummer functions) or can be easily expressed using them $(\sin z, \cos z, \sinh z, \cosh z$, Bessel functions, "incomplete" gamma-function and some other special functions).

Description of algebraic properties of the generalized hypergeometric functions and solutions of the generalized hypergeometric equations in general is still far from completion despite long history of their investigation. They are interesting for calculus in the broad sense of the word and for applications. The Siegel-Shidlovskii method (see, for example, [8]), is one of the main methods in the theory of transcendental numbers; it permits to establish the transcendency and the algebraic independence of the values of entire functions of some class that contains the functions ${ }_{l} \varphi_{q}\left(\alpha z^{q-l}\right)$ $(\alpha \in \mathbb{A})$ if these functions are algebraically independent over $\mathbb{C}(z)$.

The simplest identities between hypergeometric functions were found by Euler and Gauss. In particular, Gauss found (see, for example, [6], Sec. 6.2.2) linear equations for his hypergeometric function ${ }_{2} F_{1}$; these equations bind this function with the so-called contiguous functions. If $\varphi$ is a hypergeometric function, then the functions

$$
\varphi\left(\nu_{k} \pm\right)=\varphi\left(\nu_{1}, \ldots, \nu_{k} \pm 1, \ldots, \nu_{l} ; \lambda_{1}, \ldots, \lambda_{q} ; z\right)
$$

and $\varphi\left(\lambda_{k} \pm\right.$ ) (which are defined similarly) are said to be contiguous to $\varphi$. Functions, whose parameters differ from those of the original hypergeometric function by integer values, are said to be associated with $\varphi$.

For the vectors $\vec{\mu}=\left(\mu_{1}, \ldots, \mu_{n}\right), \vec{\eta}=\left(\eta_{1}, \ldots, \eta_{n}\right)$ we shall write $\vec{\mu} \sim \vec{\eta}$ if there exists a permutation $\pi$ of the numbers $1, \ldots, n$ such that $\mu_{i}-\eta_{\pi(i)} \in \mathbb{Z}, i=1, \ldots, n$. We shall also use the notation $\gamma \vec{\mu}+\beta=\left(\gamma \mu_{1}+\beta, \ldots, \gamma \mu_{n}+\beta\right)$, where $\gamma, \beta \in \mathbb{C}$. The expression $(\vec{\nu} ; \vec{\lambda}) \sim \gamma(\vec{\mu} ; \vec{\eta})+\beta$ means that $\vec{\nu} \sim \gamma \vec{\mu}+\beta, \vec{\lambda} \sim \gamma \vec{\eta}+\beta$. Equation 1 is said to be linearly reducible (linearly homogeneously reducible) if 
it has a solution $y \not \equiv 0$ such that $y, y^{\prime}, \ldots, y^{(q-1)}$ are linearly dependent with 1 (linearly dependent) over $\mathbb{C}(z)$, and linearly irreducible (linearly homogeneously irreducible) otherwise (see [8], Chap. 10, §1).

One can notice that contiguous functions and their derivatives are expressed as linear combinations (nonhomogeneous in general) with coefficients from $\mathbb{C}(z)$ of the functions $\varphi, \varphi^{\prime}, \ldots, \varphi^{(q-1)}$ (see [1], §§2.5, 3.7; [4], formula (19); [6], Secs. 5.2.2, 7.3.2; [8], Chap. 10, formula (47); [10], formula (12)). In $[1,4,6,8,10]$ the cases $q=l=2, \lambda_{1}=1 ; q=l, \lambda_{1}=1$; $q=2, l=\lambda_{1}=1 ; l=0 ; l=0, q / 2 \in \mathbb{N}$ are considered correspondingly. The coefficient of $\varphi\left(\nu_{k} \pm\right)$ (or $\varphi\left(\lambda_{k} \pm\right)$ ) can be identically zero for certain admissible values of the parameters of the functions. The corresponding differential equations are linearly reducible in such cases. For $\nu_{i} \notin \mathbb{Z}^{-}$, the conditions (found by Salikhov, see [7], Theorem 8) $\nu_{i}-\lambda_{j} \in \mathbb{Z}, 1 \leqslant i \leqslant l, 1 \leqslant j \leqslant q$ or the existence of a divisor $d>1$ of the numbers $l$ and $q$ such that $(\vec{\nu} ; \vec{\lambda})+1 / d \sim(\vec{\nu} ; \vec{\lambda})$ are necessary and sufficient for linear reducibility.

The author [5] has made an attempt to give a compact description of contiguity relations for arbitrary generalized hypergeometric functions (see [5], Lemma 12). However, the statement of this lemma can not be transferred to hypergeometric functions with finite radii of convergence of their power series (i. e., when $l=q$ ), and the proof in some cases was not correct. The contiguity relations can be written as $\vec{\varphi}_{1}=\Omega \vec{\varphi}+\vec{c}$, where $\vec{f}=\left(f, f^{\prime}, \ldots, f^{(q-1)}\right)^{T}, \quad \Omega \in M(q, \mathbb{C}(z)), \quad \vec{c} \in(\mathbb{C}(z))^{q}, \quad \varphi={ }_{l} \varphi_{q}(\vec{\nu} ; \vec{\lambda} ; z)$, $\varphi_{1}$ is the function $\varphi\left(\nu_{k} \pm\right)$ or $\varphi\left(\lambda_{k} \pm\right)$, the symbol $T$ means transposition. If

$$
\vec{\varphi}(\nu \pm)=\Omega_{\nu \pm} \vec{\varphi}+\vec{c}_{\nu \pm}, \quad \vec{\varphi}(\lambda \pm)=\Omega_{\lambda \pm} \vec{\varphi}+\vec{c}_{\lambda \pm},
$$

then it is easy to see that $\Omega_{\nu-}=\Omega_{\nu+}^{-1}(\nu-), \vec{c}_{\nu-}=-\Omega_{\nu-} \vec{c}_{\nu+}(\nu-), \Omega_{\lambda+}=$ $=\Omega_{\lambda-}^{-1}(\lambda+), \vec{c}_{\lambda+}=-\Omega_{\lambda+} \vec{c}_{\lambda-}(\lambda+)$. The parameter $\nu$ (and also $\lambda$ ) in this notation is arbitrarily taken from the set $\left\{\nu_{1}, \ldots, \nu_{l}\right\}$ (or $\left\{\lambda_{1}, \ldots, \lambda_{q}\right\}$ ). The symbol $\Omega_{\nu+}(\nu-)$ designates the matrix which is obtained from $\Omega_{\nu+}$ by the substitution $\nu \rightarrow \nu-1$ in its entries, where $\nu$ is the chosen parameter. The symbols $\Omega_{\lambda-}(\lambda+), \vec{c}_{\nu+}(\nu-), \vec{c}_{\lambda-}(\lambda+)$ are defined similarly.

2. Main results. We present an algorithm for finding contiguity relations and also their generalizations to the case of associated functions and solution matrices $\Phi_{i}$ of their corresponding homogeneous differential equations $L\left(\vec{\nu}_{i} ; \vec{\lambda}_{i} ; z\right) y=0$ in the proof of the following theorem. The matrix $\Phi$ for the equation $L(\vec{\nu} ; \vec{\lambda} ; z) y=0$ is a solution matrix of the corresponding first order vector equation. In this theorem we also solve the 
problem of cogradience (see definition in [3], Sec. 1) of the hypergeometric equations, whose parameters differ by integer values.

Theorem 1. Suppose that $\vec{\nu}_{i} \in \mathbb{C}^{l}, \vec{\lambda}_{i} \in\left(\mathbb{C} \backslash \mathbb{Z}^{-}\right)^{q}, q \geqslant \max (2, l)$, $\varphi_{i}={ }_{l} \varphi_{q}\left(\vec{\nu}_{i} ; \vec{\lambda}_{i} ; z\right)$, the equation $L\left(\vec{\nu}_{i} ; \vec{\lambda}_{i} ; z\right) y=0$ is linearly homogeneously irreducible, $\Phi_{i}$ is an arbitrary solution matrix of this equation, for $i=1,2$, and $\left(\vec{\nu}_{1} ; \vec{\lambda}_{1}\right) \sim\left(\vec{\nu}_{2} ; \vec{\lambda}_{2}\right)$. Then there exist matrices

$$
\Omega \in G L\left(q, \mathbb{C}\left[z^{ \pm 1},(1-z)^{-\varepsilon}\right]\right), \quad C \in G L(q, \mathbb{C})
$$

and a vector $\vec{c} \in\left(\mathbb{C}\left[z^{ \pm 1},(1-z)^{-\varepsilon}\right]\right)^{q}, \varepsilon=\delta_{q}^{l}$ such that

$$
\vec{\varphi}_{1}=\Omega \vec{\varphi}_{2}+\vec{c}, \quad \Phi_{1}=\Omega \Phi_{2} C .
$$

The proof of the Theorem 1 requires the following lemma.

Lemma 1. [5, lemma 1] The function (or, for $l>q$, the formal power series) $z^{\gamma}{ }_{l} \varphi_{q}\left(\vec{\nu} ; \vec{\lambda} ; \alpha z^{p}\right)$, where $\vec{\nu} \in \mathbb{C}^{l}, \vec{\lambda} \in\left(\mathbb{C} \backslash \mathbb{Z}^{-}\right)^{q}, \quad \alpha, \gamma \in \mathbb{C}, l, q \in \mathbb{Z}^{+}$, $l+q \neq 0, p \in \mathbb{Z}$, satisfies the differential equation

$$
\left(\prod_{i=1}^{q}\left(\delta+p\left(\lambda_{i}-1\right)-\gamma\right)-\alpha p^{q-l} z^{p} \prod_{i=1}^{l}\left(\delta+p \nu_{i}-\gamma\right)\right) y=p^{q} z^{\gamma} \prod_{i=1}^{q}\left(\lambda_{i}-1\right),
$$

the empty product of brackets equals 1 .

Corollary 1. If $\lambda_{i}-\lambda_{k} \notin \mathbb{Z}, i \neq k$, and $q \geqslant \max (1, l)$, then the functions

$$
z^{\left(1-\lambda_{k}\right) p}{ }_{l} F_{q-1}\left(\vec{\nu}+1-\lambda_{k} ; \vec{\lambda}+1-\lambda_{k} ; \alpha z^{p}\right), \quad k=1, \ldots, q
$$

constitute the fundamental system of solution of the equation

$$
L\left(\vec{\nu} ; \vec{\lambda} ; \alpha z^{p}\right) y=0
$$

where

$$
L\left(\vec{\nu} ; \vec{\lambda} ; \alpha z^{p}\right) \equiv \prod_{i=1}^{q}\left(\delta+p\left(\lambda_{i}-1\right)\right)-\alpha p^{q-l} z^{p} \prod_{i=1}^{l}\left(\delta+p \nu_{i}\right)
$$

is obtained from $L(\vec{\nu} ; \vec{\lambda} ; z) y=0$ by the substitution $z \rightarrow \alpha z^{p}$.

Proof of the Corollary 1. By the Lemma 1, the functions

$$
z^{\left(1-\lambda_{k}\right) p} \varphi_{q}\left(\vec{\nu}+1-\lambda_{k} ; \vec{\lambda}+1-\lambda_{k} ; \alpha z^{p}\right), \quad k=1, \ldots, q,
$$


satisfy the equation $L\left(\vec{\nu} ; \vec{\lambda} ; \alpha z^{p}\right) y=0$, are linearly independent over $\mathbb{C}$, and can be written as (2), because the $k$-th component of the vector $\vec{\lambda}+1-\lambda_{k}$ equals 1 .

As it follows from the Lemma 1 , the equation $L\left(\vec{\nu} ; \vec{\lambda} ; \alpha z^{p}\right) y=0$ for $q \geqslant l$ has the form

$$
\begin{gathered}
\left(1-\alpha z^{p}\right)^{\varepsilon} z^{q} y^{(q)}+\left(\left(\lambda_{1}+\cdots+\lambda_{q}-q\right) p+(q-1) \frac{q}{2}-\varepsilon_{1} \alpha p z^{p}-\right. \\
\left.-\varepsilon \alpha\left(\left(\nu_{1}+\cdots+\nu_{q}\right) p+(q-1) \frac{q}{2}\right) z^{p}\right) z^{q-1} y^{(q-1)}+ \\
+\cdots+p^{q}\left(\left(\lambda_{1}-1\right) \ldots\left(\lambda_{q}-1\right)-\alpha \nu_{1} \ldots \nu_{l} z^{p}\right) y=0,
\end{gathered}
$$

where $\varepsilon=\delta_{q}^{l}, \varepsilon_{1}=\delta_{q}^{l+1}$. The Wronskian of this equation equals

$$
W=c z^{-\left(\lambda_{1}+\cdots+\lambda_{q}-q\right) p-(q-1) q / 2}\left(1-\alpha z^{p}\right)^{\left(\lambda_{1}+\cdots+\lambda_{q}-\nu_{1} \cdots-\nu_{q}-q\right) \varepsilon} e^{\alpha z^{p} \varepsilon_{1}},
$$

where $c \in \mathbb{C} \backslash\{0\}$.

Proof of the Theorem 1. We start with the following remark. Suppose that $\Psi$ is a solution matrix of some linear homogeneous system $\vec{y}^{\prime}=A \vec{y}$, $\Omega=\Omega(z)$ and $C$ are arbitrary non-singular matrices of the same size as $A=A(z), C$ does not depend on $z$. Elementary calculations show that $\Psi_{1}=\Omega \Psi C$ is a solution matrix of the system $\vec{y}^{\prime}=A_{1} \vec{y}$, where $A_{1}=\left(\Omega^{\prime}+\Omega A\right) \Omega^{-1}$. On the other hand, if $\Psi_{1}$ is a solution matrix of the system $\vec{y}^{\prime}=A_{1} \vec{y}$, then, setting $\Psi_{2}=\Omega^{-1} \Psi_{1}$, we obtain

$$
\Psi_{2}^{\prime}=\left(\Omega^{-1} \Psi_{1}\right)^{\prime}=-\Omega^{-1} \Omega^{\prime} \Omega^{-1} \Psi_{1}+\Omega^{-1} A_{1} \Psi_{1}=A \Omega^{-1} \Psi_{1}=A \Psi_{2},
$$

whence $\Psi_{2}=\Psi C$ and $\Psi_{1}=\Omega \Psi C$. If $\vec{\psi}, \vec{\psi}_{1}$ are arbitrary solutions of linear systems $\vec{y}^{\prime}=A \vec{y}+\vec{c}$ and $\vec{y}^{\prime}=A_{1} \vec{y}+\vec{c}_{1}$ respectively, where $\vec{\psi}_{1}=\Omega \vec{\psi}+\vec{c}_{2}$, then we obtain $A_{1}=\left(\Omega^{\prime}+\Omega A\right) \Omega^{-1}$ again. Hence, the equality $\vec{\psi}_{1}=\Omega \vec{\psi}+\vec{c}_{2}$ for some solutions of initial systems implies the equality $\Psi_{1}=\Omega \Psi C$ for arbitrary solution matrices of the homogeneous systems that correspond to the initial ones. If $\Omega \in M(q, \mathbb{C}(z)), \vec{c}, \vec{c}_{1}, \vec{c}_{2} \in(\mathbb{C}(z))^{q}$, then in the case of linear irreducibility (or linear homogeneous irreducibility of the corresponding homogeneous systems) matrices $\Omega$ and $A_{1}$ are determined uniquely, and $\Omega$ is always non-singular.

To prove the theorem, it suffices to restrict ourselves to the case in which $\nu_{1, j}=\nu_{2, j}=\nu_{j}, \quad \lambda_{1, t}=\lambda_{2, t}=\lambda_{t}$ for all parameters except the one whose values differ by 1 . Assume that this parameter is $\nu_{k}, 1 \leqslant k \leqslant l$. 
Suppose that $\nu_{k}=\nu_{2, k}=\nu_{1, k}-1$. Differentiating the identity

$$
\nu_{k} \varphi_{2}\left(\nu_{k}+\right)=\left(\delta+\nu_{k}\right) \varphi_{2}=\nu_{k} \varphi_{2}+z \varphi_{2}^{\prime}
$$

(see [6], Sec. 5.2.2), $q-1$ times, we find

$$
\nu_{k} \vec{\varphi}_{1}=\bar{\Omega} \vec{\varphi}_{2}+\left(0, \ldots, 0, b z^{1-q}(1-z)^{-\varepsilon}\right)^{T},
$$

where $b=\left(\lambda_{1}-1\right) \ldots\left(\lambda_{q}-1\right), \quad \bar{\Omega}$ is such a matrix that its $j$-th row for $j \neq q$ contains only two nonzero entries: $a_{j, j}=\nu_{k}+j-1$ and $a_{j, j+1}=z$, while the entries in the $q$-th row are already obtained from the coefficients of the equation $L\left(\vec{\nu}_{2} ; \vec{\lambda}_{2} ; z\right) y=b$ and, by (3), belong to $\mathbb{C}\left[z^{ \pm 1},(1-z)^{-\varepsilon}\right]$. From the linear irreducibility of the equations under consideration the inequalities $\nu_{k} \neq 0$ and $|\bar{\Omega}| \not \equiv 0$ follow. Aforesaid means that $\Phi_{1}=\bar{\Omega} \Phi_{2} C$, and from here, taking equality (4) and inclusion $|\bar{\Omega}| \in \mathbb{C}\left[z^{ \pm 1},(1-z)^{-\varepsilon}\right]$ into account, we obtain $|\bar{\Omega}|=a z^{n}(1-z)^{m \varepsilon}, a \in \mathbb{C} \backslash\{0\}, n, m \in\{0,1,-1\}$, $\bar{\Omega} \in G L\left(q, \mathbb{C}\left[z^{ \pm 1},(1-z)^{-\varepsilon}\right]\right)$.

From here, in the case of an increase (and, hence, also a decrease) of $\nu_{k}$ by 1 , the assertion of the theorem holds.

If $\nu_{1, j}=\nu_{2, j}=\nu_{j}, \quad \lambda_{1, t}=\lambda_{2, t}=\lambda_{t}$ for all parameters except $\lambda_{k}$, $1 \leqslant k \leqslant q$, similar arguments and the identity

$$
\left(\lambda_{k}-1\right) \varphi\left(\lambda_{k}-\right)=\left(\lambda_{k}-1\right) \varphi+z \varphi^{\prime}
$$

are used. The theorem is proved.

3. Examples. We obtain

$$
\Omega_{\nu+}=\frac{1}{\nu}\left(\begin{array}{cc}
\nu & z \\
\nu & \nu-\lambda+1+z
\end{array}\right) ; \quad \Omega_{\lambda-}=\frac{1}{\lambda-1}\left(\begin{array}{cc}
\lambda-1 & z \\
\nu & z
\end{array}\right)
$$

by the algorithm presented in the proof of the Theorem 1 for Kummer functions

$$
A_{\lambda, \nu}(z)={ }_{1} \varphi_{2}(\nu ; 1, \lambda ; z)={ }_{1} F_{1}\left(\begin{array}{c}
\nu \\
\lambda
\end{array} \mid z\right)=\sum_{n=0}^{\infty} \frac{(\nu)_{n}}{n !(\lambda)_{n}} z^{n},
$$

while for Gauss hypergeometric functions

$$
{ }_{2} \varphi_{2}(\nu, \mu ; 1, \lambda ; z)={ }_{2} F_{1}\left(\begin{array}{c}
\nu, \mu \\
\lambda
\end{array} \mid z\right)=\sum_{n=0}^{\infty} \frac{(\nu)_{n}(\mu)_{n}}{n !(\lambda)_{n}} z^{n}
$$


we obtain, similarly,

$$
\begin{gathered}
\Omega_{\nu+}=\frac{1}{\nu(1-z)}\left(\begin{array}{cc}
\nu(1-z) & z(1-z) \\
\nu \mu & \nu-\lambda+1+\mu z
\end{array}\right) ; \\
\Omega_{\lambda-}=\frac{1}{(\lambda-1)(1-z)}\left(\begin{array}{cc}
(\lambda-1)(1-z) & z(1-z) \\
\nu \mu & (\nu+\mu-\lambda+1) z
\end{array}\right) .
\end{gathered}
$$

The proved Theorem 1 can be easily generalized to the case of functions ${ }_{l} \varphi_{q}\left(\vec{\nu} ; \vec{\lambda} ; \alpha z^{p}\right)$ by means of the substitution $z \rightarrow \alpha z^{p}$. Here $\alpha \in \mathbb{C}, p \in \mathbb{N}$. For example, for functions

$$
K_{\lambda, \mu}(z)={ }_{0} \varphi_{2}\left(\lambda+1, \mu+1 ;-z^{2} / 4\right)=\sum_{n=0}^{\infty} \frac{(-1)^{n}}{(\lambda+1)_{n}(\mu+1)_{n}}\left(\frac{z}{2}\right)^{2 n},
$$

introduced by Shidlovskii (see [8], Chap. 6, §5]), we obtain

$$
\Omega_{\lambda-}=\frac{1}{2 \lambda z}\left(\begin{array}{cc}
2 \lambda z & z^{2} \\
-z^{2}-4 \lambda \mu & -2 \mu z
\end{array}\right) ; \quad \vec{c}_{\lambda-}=\frac{2 \mu}{z}\left(\begin{array}{l}
0 \\
1
\end{array}\right)
$$

\section{References}

[1] Andrews G., Askey R., Roy R. Special Functions. Cambridge University Press, 2000; Moscow: MCNMO, 2013.

[2] Bateman H., Erdélyi A. Higher transcendental functions. Vol. 1. The hypergeometric function, Legendre functions. New York-Toronto-London: McGraw-Hill, 1953; Moscow: Nauka, 1965.

[3] Beukers F., Brownawell W. D., Heckman G. Siegel normality. Annals of Math., 1988, vol. 127, pp. 279-308.

[4] Bytev V. V., Kalmykov M. Yu., Kniehl B. A. HYPERDIRE, HYPERgeometric functions DIfferential REduction: MATHEMATICA-based packages for differential reduction of generalized hypergeometric functions ${ }_{p} F_{p-1}, F_{1}, F_{2}, F_{3}, F_{4}$. Computer Physics Communications, 2013, vol. 184, pp. 2332-2342.

[5] Gorelov V. A. On the algebraic independence of values of generalized hypergeometric functions. Mathematical Notes, 2013, vol. 94, no. 1, pp. 82-95. DOI: https://doi.org/10.1134/S0001434613070080

[6] Luke Y. Mathematical functions and their approximations. New York: Academic Press, 1975; Moscow: Mir, 1980. 
[7] Salikhov V. Kh. Formal solutions of linear differential equations and their application in the theory of transcendental numbers. Trans. Moscow Math. Soc., 1989, pp. $219-251$.

[8] Shidlovskii A. B. Transcendental numbers. Moscow: Nauka, 1987; BerlinNew York: Walter de Gruyter, 1989.

[9] Slater L. J. Generalized Hypergeometric Functions. London-New York: Cambridge University Press, 1966.

[10] Viskina G. G., Salikhov V. Kh. Algebraic relations between hypergeometric E-function and its derivatives. Mathematical Notes, 2002, vol. 71, no. 6, pp. 761-772. DOI: https://doi.org/10.1023/A:1015864711107

Received March 4, 2018.

In revised form, September 12, 2018.

Accepted September 13, 2018.

Published online October 15, 2018.

National Research University «Moscow Power Engineering Institute»

14 Krasnokazarmennaya str., Moscow 111250, Russia

E-mail: gorelov.va@mail.ru 